\title{
Energy Harvestable from a Colloidal Vehicle Suspension
}

\author{
Barenten Suciu ${ }^{\text {a, }}$, Keisuke Koyanagi ${ }^{\text {b }}$ \\ ${ }^{a}$ Department of Intelligent Mechanical Engineering, Fukuoka Institute of Technology, \\ 3-30-1 Wajiro-Higashi, Higashi-ku, Fukuoka 811-0295, Japan \\ ${ }^{\mathrm{b}}$ Graduate School of Engineering, Fukuoka Institute of Technology, \\ 3-30-1 Wajiro-Higashi, Higashi-ku, Fukuoka 811-0295, Japan \\ *Corresponding Author: suciu@fit.ac.jp
}

\begin{abstract}
Vehicle suspensions able to harvest electrical energy are usually using electromagnetic motor and generators. Since the bound-rebound translation has to be transformed into rotation, by employing various mechanisms (ball screw and nut; rack and gear; hydraulic cylinder and motor, etc.), such systems are relatively complex, costly and unreliable. Alternatively, electromagnetic induction and piezoelectricity were used to produce electrical power. Such systems are cheaper and simpler, since there is no need to transform the motion. In this work, a piezoelectric stack is placed inside the cylinder of a colloidal damper. Working colloid produces a cyclical compression-decompression on the surface of the piezoelectric disks, and in this way mechanical energy recovered from the rough road excitation can be partially transformed into electrical energy. Piezoelectric stack is serially mounted together with a helical spring in order to obtain a vehicle suspension able to support both compressive and tensile loads. From excitation tests carried on a ballscrew shaker, one determines the amount of harvestable power. Although the obtained power $(1-100 \mathrm{~W})$ is relatively modest, some applications such as, charge and recharge of a battery, or usage as electrical power source for the actuator of a fuel injection system can be considered.
\end{abstract}

Keywords: piezoelectric device, damper, intelligent vehicle suspension, energy conversion.

\section{Introduction}

Along with the development of a new generation of electrical and hybrid vehicles, increasingly amounts of electrical power are required for the transmission system, for driving various motors, for operating sensors, etc. E.g., a voltage of 288/202 V (DC) and an electrical energy of $1.8 / 1.3 \mathrm{kWh}$ are required by the main battery of recently developed hybrid vehicles ${ }^{(1)}$. By the way, to open/close the fuel injection system, trigger voltage of the piezoelectric actuator employed should be adjusted in the range of 220 to $260 \mathrm{~V}$, for fuel pressures varying in the higher range of 150 to $170 \mathrm{MPa}$, and in the range of 50 to $80 \mathrm{~V}$, for fuel pressures varying in the lower range of 30 to $55 \mathrm{MPa}^{(2,3)}$. Relative to such power demand, the harvestable electrical power is on order of several $\mathrm{kW}$ in the case of regenerative breaking systems, in the range of $0.2-1 \mathrm{~kW}$ for harvesting from thermal systems, and in the range of $0.1-0.2 \mathrm{~kW}$ in the case of solar panels ${ }^{(4)}$. However, the subject of harvestable energy from the vehicle suspension is still open to debate.

A vehicle suspension able to generate electrical power transforms a part of the kinetic energy of bound-rebound motion excited by the road roughness into electrical energy. Mainly, two types of systems have been developed. Thus, if an electromagnetic motor and generator is used to produce electricity, the translational bound-rebound motion has to be transformed into rotational motion of the shaft, by using an adequate mechanism such as: ball screw and nut ${ }^{(5)}$, hydraulic cylinder and motor $^{(6)}$, rack and spur gear ${ }^{(7)}$, etc. Such systems are costly, complex from a structural standpoint, and have relatively low efficiency, robustness and reliability. On the other hand, if electromagnetic induction ${ }^{(8)}$ and piezoelectric elements $^{(9-11)}$ are used to produce electricity, there is no need to transform the bound-rebound movement. Such systems are relatively cheaper and simpler from a structural standpoint.

Thus, piezoelectric device might be introduced inside the air bag of a pneumatic suspension ${ }^{(9)}$ (Fig. 1) or inside the cylinder of an oil damper ${ }^{(9)}$ (left side of Fig. 2) or inside the cylinder of a colloidal damper ${ }^{(10,11)}$ (right side of Fig. 2). Under the rough road excitation, the working fluid undergoes 
cyclical compression-decompression, and since such dynamic pressure acts on the surface of piezoelectric device electrical power is produced. In this work, the energy harvestable from a colloidal vehicle suspension is evaluated. A filter is used to divide the cylinder into a room of constant volume which accommodates silica grains, and a room of variable volume in which only water is supplied ${ }^{(12,13)}$. Water is able to freely pass, but silica grains are unable to pass through the filter.

Under the particular design shown in Fig. 2, colloidal damper's life was extended up to the requirements imposed to usual vehicle suspensions ${ }^{(14)}$.

Piezoelectric stack can be introduced inside the room of constant volume, in which case the working liquid is the colloidal solution consisted of water and silica particles.

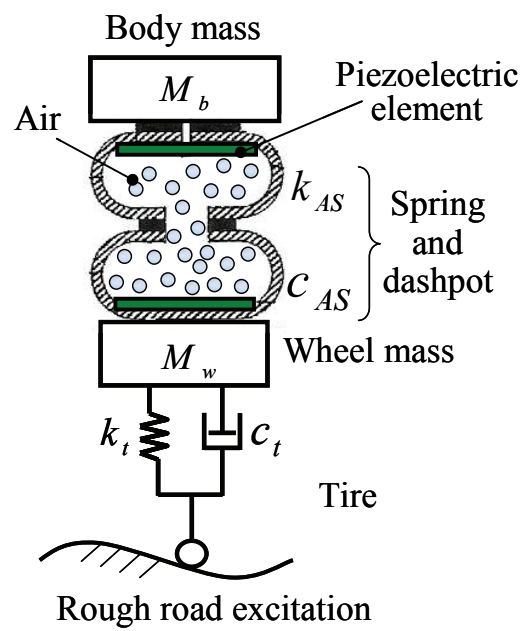

Fig. 1. Vehicle suspension able to generate electrical power by using piezoelectric elements compressed by air ${ }^{(9)}$.

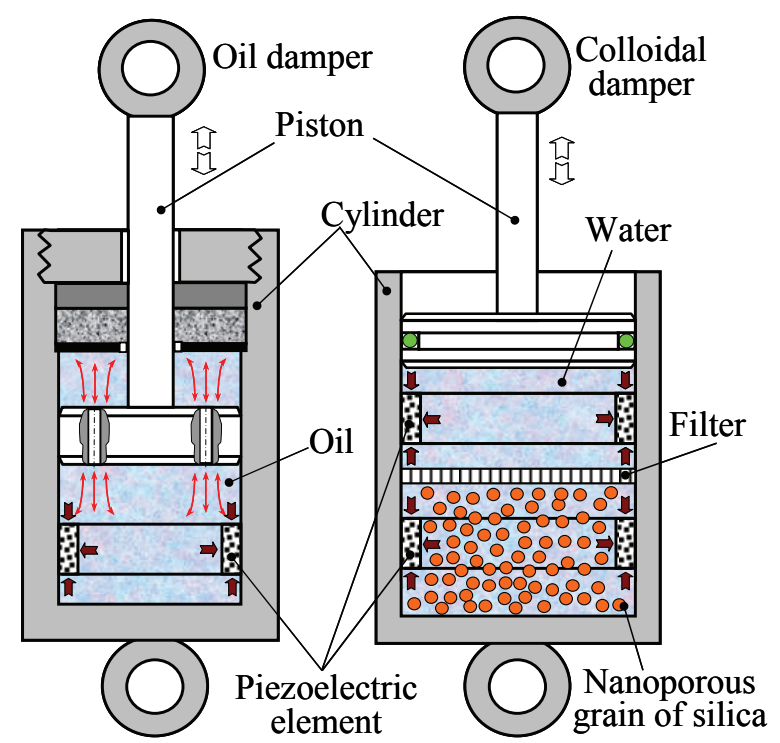

Fig. 2. Vehicle suspension able to generate electrical power by using piezoelectric elements compressed by oil ${ }^{(9)}$, water $^{(10)}$, or colloidal mixture of water and silica grains ${ }^{(10)}$.
Alternatively, piezoelectric stack can be placed inside the room of variable volume, in which case the working liquid is water. In this work, the piezoelectric stack is serially mounted together with a spring and such ensemble is pre-compressed inside the cylinder. For this reason, the colloidal suspension is able to support both compressive and tensile loads. From excitation tests carried on a ball-screw shaker one estimates the energy harvestable and evaluates the feasibility of the proposed suspension to accommodate practical applications.

\section{Generated Voltage and Electrical Power}

A piezoelectric device able to produce electrical power when compressed by axial pressure on the end flat surfaces and, simultaneously pressurized by a radial pressure on the inner cylindrical surface of its tubular body is shown in Fig. 3. Geometrical features, generated voltage and electrical power, as well as design conditions to achieve sufficient strength for the piezoelectric device are shown in Table 1. If $n$ piezoelectric devices are electrically connected in parallel the obtained power $P$ can be calculated as (see Table 1):

$$
P=0.5 \varepsilon_{0} K_{z} n V p^{2} f\left[g_{z z}^{2}+g_{z r}^{2}\left(1-d_{i} / d_{o}\right)^{-2}\right] .
$$

To achieve proper design of the colloidal suspension, the voltage generated due to the action of the axial pressure should equal the voltage generated due to the action of the radial pressure. Under such design condition, ratio of the inner diameter to the outer diameter can be calculated as:

$$
d_{i} / d_{o}=1-g_{z r} / g_{z z}
$$

Thus, for a certain ceramic material with piezoelectric constants $g_{z z}=26.1 \mathrm{mV} \cdot \mathrm{m} / \mathrm{N}$ and $g_{z r}=11.9 \mathrm{mV} \cdot \mathrm{m} / \mathrm{N}$, ratio of the inner diameter to the outer diameter becomes 0.544 .

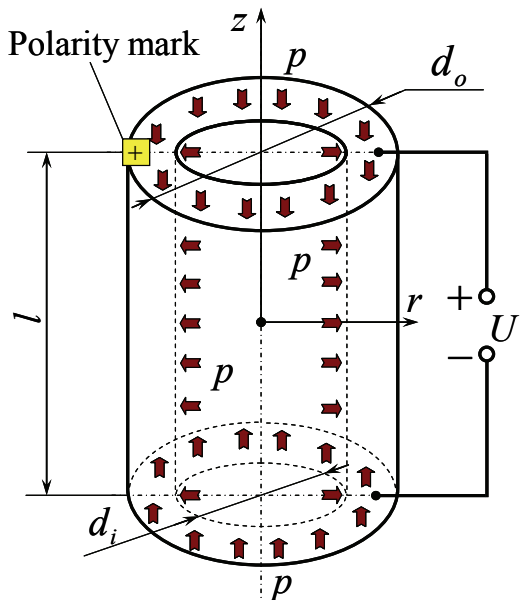

Fig. 3. Tubular piezoelectric element axially and radially loaded by uniformly applied pressure. 
Table 1. Performances of the tubular piezoelectric device.

\begin{tabular}{|c|c|c|}
\hline & Axial pressure & Radial pressure \\
\hline$d_{i}=0$ & $\begin{array}{c}\text { Circular bar } \\
\text { Circular disk }\end{array}$ & $\begin{array}{c}\text { Circular bar } \\
\text { Circular disk }\end{array}$ \\
\hline $0<d_{i}<d_{o}$ & $\begin{array}{c}\text { Annular bar } \\
\text { Annular disk }\end{array}$ & $\begin{array}{c}\text { Annular bar } \\
\text { Annular disk }\end{array}$ \\
\hline Volume[m $\left.{ }^{3}\right]$ & \multicolumn{2}{|c|}{$V=0.25 \pi\left(d_{o}^{2}-d_{i}^{2}\right) l$} \\
\hline Voltage [V] & $U=g_{z z} l p$ & $U=\frac{g_{z r} l p}{1-d_{i} / d_{o}}$ \\
\hline $\begin{array}{c}\text { Power [W] } \\
\text { Design } \\
\text { conditions }\end{array}$ & $P=\frac{\varepsilon_{0} g_{z z}^{2} K_{z} n V p^{2} f}{2}$ & $P=\frac{\varepsilon_{0} g_{z r}^{2} K_{z} n V p^{2} f}{2\left(1-d_{i} / d_{o}\right)^{2}}$ \\
\hline & $p_{\max } \leq \sigma_{a}$ & $\frac{1}{3} \leq \frac{d_{i}}{d_{o}} \leq \sqrt{\frac{\sigma_{a}-p_{\max }}{\sigma_{a}+p_{\max }}}$ \\
\hline
\end{tabular}

Pressurizing the piezoelectric device along both axial and radial directions, under the design condition shown by Eq. (2), a double amount of electrical power can be generated. Further, for a four-wheel vehicle, under the assumption that all four suspensions are working at the same instantaneous pressure, Eq. (1) can be rewritten as:

$$
P=4 \varepsilon_{0} K_{z} g_{z z}^{2} n V p^{2} f .
$$

Substituting Eq. (2) into expression for the volume of the piezoelectric device (see Table 1), and then, substituting the result obtained into Eq. (3), one finds the total electrical power harvestable from the four-wheel vehicle as:

$$
P=\pi \varepsilon_{0} K_{z} g_{z r}\left(2 g_{z z}-g_{z r}\right) n d_{o}^{2} l p^{2} f .
$$

\section{Test Rig and Experimental Procedure}

Test rig employed during excitation tests of the proposed harvesting colloidal damper is shown in Fig. 4. The made-in-house ball-screw shaker is consisted of a ballscrew mechanism driven by an electrical motor via a belt transmission, a controller and a power unit. For safety reasons contact limit-switches are used. Excitation frequency can be changed by varying the rotational speed of the ball-screw. Axial force $F[\mathrm{~N}]$ is measured by using a load-cell placed in between the piston-rod of the colloidal suspension and the rod of the ball-screw. Piston stroke $S[\mathrm{~mm}]$ is measured by using a laser displacement sensor (see Fig. 5).

It is well-known the fact that piezoelectric effect cannot be obtained under tension loading in the case of traditional piezoelectric stacks.

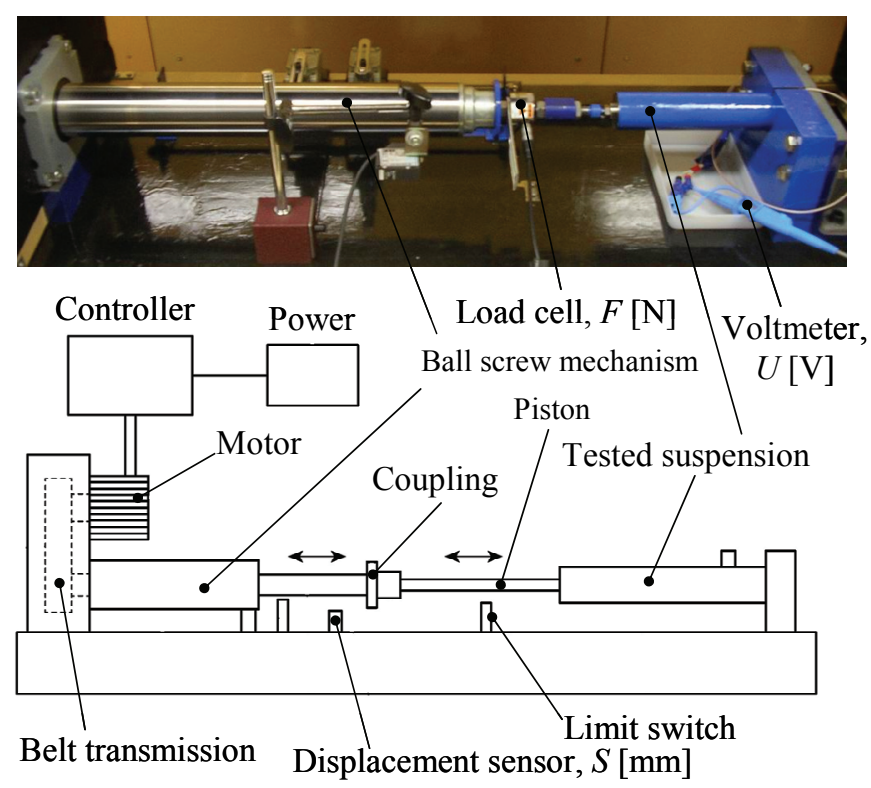

Fig. 4. Schematic view and photograph of the test rig.

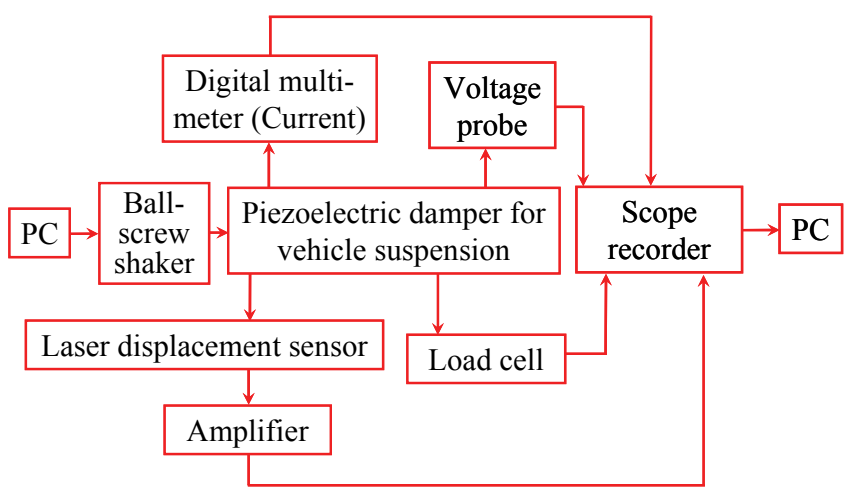

Fig. 5. Loading and measurement equipment.

However, in the case of the proposed suspension, the piezoelectric stack is serially mounted together with a helical compression spring. Since the helical spring is precompressed by a force of $6 \mathrm{kN}$, such vehicle suspension is able to support both compressive and tensile loads. Nevertheless, the maximum allowable tension force is limited to $6 \mathrm{kN}$. From excitation tests carried on the above mentioned ball-screw shaker, one evaluates the amount of electrical energy harvestable from the proposed suspension.

\section{Experimental Results and Discussions}

Figure 6 illustrates the variation of the hysteresis loop and Fig. 7 shows the variation of the working pressure during one period, for the following values of the maximum pressure of the colloidal suspension: 20, 25, 30, 35, 40 and 60MPa. In the case of a classical vehicle suspension, pressure of the working fluid inside the cylinder of an oil damper or inside 
the air bag of a pneumatic suspension varies in the range of 0 to $10 \mathrm{MPa}$. However, in the case of a colloidal suspension, pressure inside the cylinder varies in the range of 20 to $60 \mathrm{MPa}$ (see Figs. 6 and 7), i.e., the maximum pressure is 6 times higher, and the pressure variation range is 4 times wider. Thus, a substantially increased harvesting effect can be obtained, due to the fact that according to Eq. (4) the electrical power production proportionally varies with the second power of the pressure change. Figure 8 illustrates the variation of the dissipated energy $E$ of the colloidal suspension against the maximal working pressure.

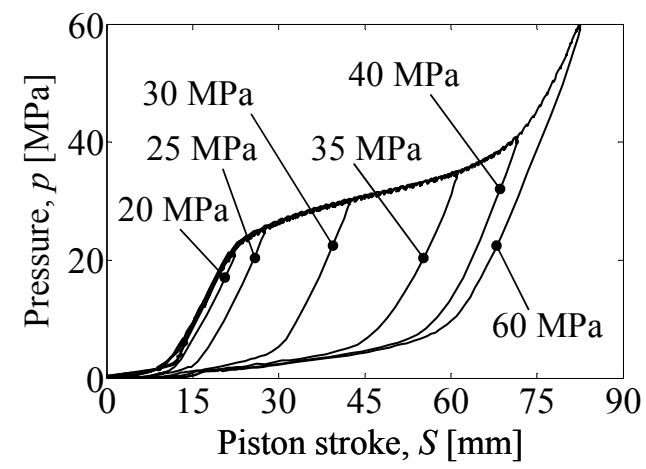

Fig. 6. Variation of the hysteresis loop of a colloidal suspension against the maximum applied pressure.

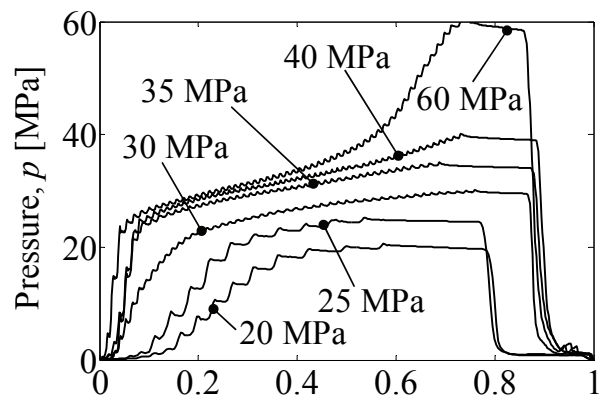

Dimensionless time, $t f[-]$

Fig. 7. Pressure variation during a complete working cycle of the colloidal suspension, recorded for various values of the maximum applied pressure.

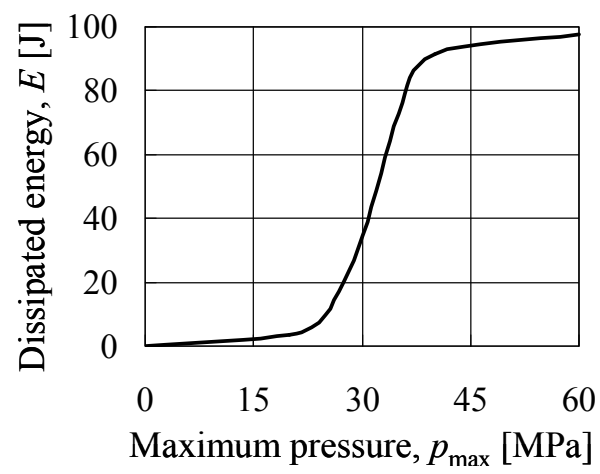

Fig. 8. Variation of the dissipated energy versus the maximum applied pressure.
Dissipated energy is calculated by multiplying the cross-sectional area of the piston with the area of the hysteresis loop in the $(S, p)$ system of coordinates (Fig. 6).

One observes that the dissipated energy monotonically increases at augmentation of the maximum working pressure.

Figure 9 presents the variation of the mean pressure $p_{m}$ of the colloidal suspension versus the maximum working pressure. Mean pressure is defined as the average value on the graph $p=p(t f)$ illustrated in Fig. 7, i.e., the value obtained by integrating the working pressure versus the dimensionless time over one period.

One observes that the mean pressure monotonically increases at augmentation of the maximum working pressure.

Figure 10 illustrates the variation of the mean generated voltage versus maximum working pressure of the colloidal damper, for various values of the thickness $(l=0.1$, 0.7 and $1.0 \mathrm{~mm}$ ) of the piezoelectric device.

Such results were obtained as follows. For a certain given maximum working pressure the mean pressure can be calculated as illustrated in Fig. 9.

Then, by substituting the calculated mean pressure, the piezoelectric constant of the generated voltage $g_{z z}=$ $26.1 \mathrm{mV} \cdot \mathrm{m} / \mathrm{N}$ and, the thickness of the piezoelectric device $l$ $=0.1,0.7$ and $1.0 \mathrm{~mm}$ into the equation of the generated voltage $U=g_{z z} l p$ (see Table 1), the mean value $U_{m}$ of the generated voltage can be calculated for various values of the thickness of the piezoelectric device.

Figure 11 illustrates the variation of the mean generated electrical power versus maximum working pressure of the colloidal damper, for various values of the thickness of the piezoelectric device.

Such results were obtained as follows. For a certain given maximum working pressure the mean pressure can be calculated as illustrated in Fig. 9.

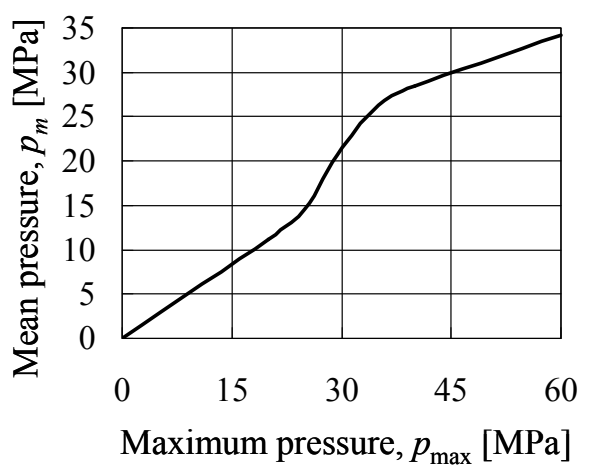

Fig. 9. Variation of the mean pressure calculated during a complete working cycle versus the maximum pressure. 


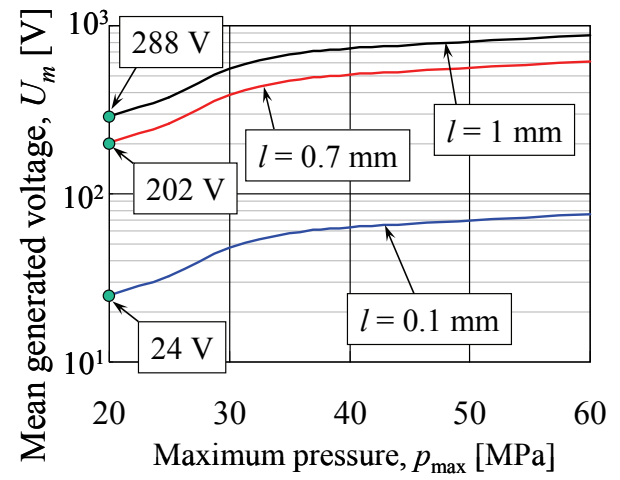

Fig. 10. Variation of the mean generated voltage versus the maximum applied pressure for various disk thicknesses.

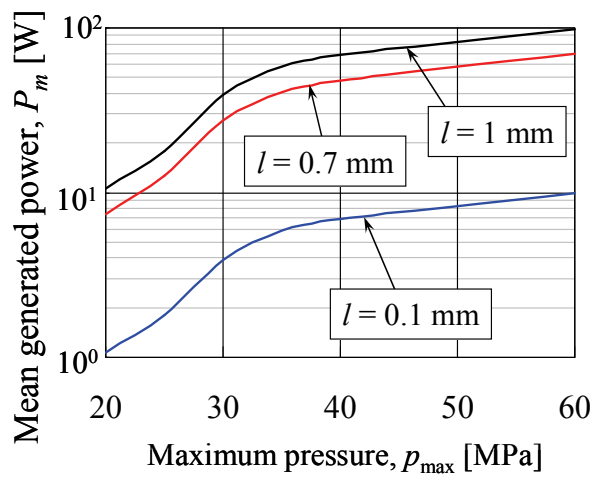

Fig. 11. Variation of the mean generated power versus the maximum applied pressure for various disk thicknesses.

Then, by substituting the value of the mean pressure, the number of piezoelectric devices $n=55$, the frequency $f$ $=10 \mathrm{~Hz}$, the outer diameter of the piezoelectric device $d_{o}=$ $60 \mathrm{~mm}$, the piezoelectric constants of the generated voltage $g_{z z}=26.1 \mathrm{mV} \cdot \mathrm{m} / \mathrm{N}$ and $g_{z r}=11.9 \mathrm{mV} \cdot \mathrm{m} / \mathrm{N}$, the relative dielectric constant of the piezoelectric device $K_{z}=3,200$, the absolute dielectric constant $\varepsilon_{0}=8.85 \mathrm{pF} / \mathrm{m}$, and the thickness $(l=0.1,0.7,1.0 \mathrm{~mm})$ of the piezoelectric device into the Eq. (4), the mean value $P_{m}$ of the generated electrical power can be calculated.

From Fig. 11 one observes that in order to achieve a mean generated voltage of $U_{m}=12 \mathrm{~V}$ (usual voltage of a battery equipping an automobile propelled by an internal combustion engine), the necessary thickness of the piezoelectric device is too small, and consequently, the proposed harvesting colloidal suspension is unlikely to be used in practical applications. However, in order to achieve a mean generated voltage of $U_{m}=24 \mathrm{~V}$ (usual voltage of a battery equipping a truck propelled by an internal combustion engine), a mean generated voltage of $U_{m}=202$ $\mathrm{V}$ and $U_{m}=288 \mathrm{~V}$ (voltage of the main battery of a hybrid vehicle), the necessary thickness of the piezoelectric device $(l=0.1,0.7,1.0 \mathrm{~mm})$ can be technologically obtained in a relatively easy manner. Thus, the harvesting colloidal damper is likely to be used in such practical applications.

Furthermore, from Fig. 11 one observes that the mean electrical power harvested by the proposed colloidal damper varies in the range of 1 to $100 \mathrm{~W}$. Although the power produced is relatively modest, some practical applications such as, charge and recharge of a battery, or usage as electrical power source for the actuator of a fuel injection system can be considered.

Next, Fig. 12 illustrates the variation of the damping force $F$ versus the excitation time $t$, and Fig. 13 presents the variation of the generated voltage $U$ against the excitation time, during tests at a frequency $f=8 \mathrm{~Hz}$. The proposed colloidal suspension was tested for loading both toward the extension and compression directions.

Such experimental results confirm the fact that the piezoelectric stack of the proposed harvesting colloidal damper works both under compressive and tensile loads. One observes that under the same working conditions concerning the amplitude and frequency of vibration, the peak-to-peak force and voltage obtained during extension are larger than those obtained during compression.

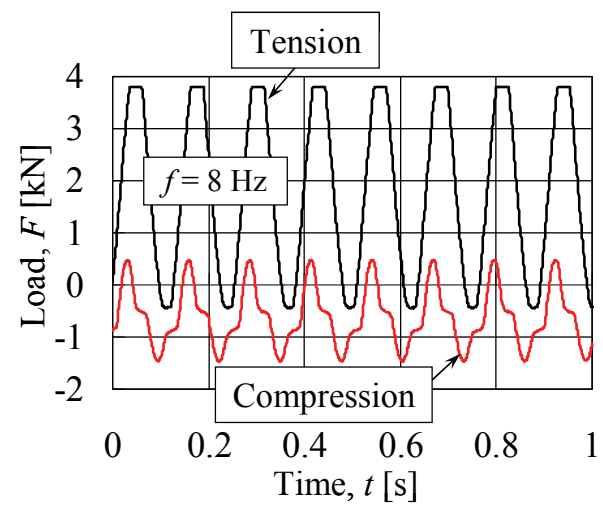

Fig. 12. Variation of the load versus the excitation time.

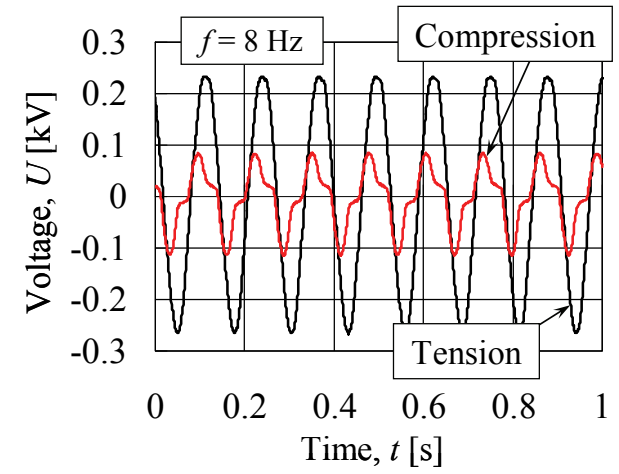

Fig. 13. Variation of the mean generated voltage versus the excitation time. 


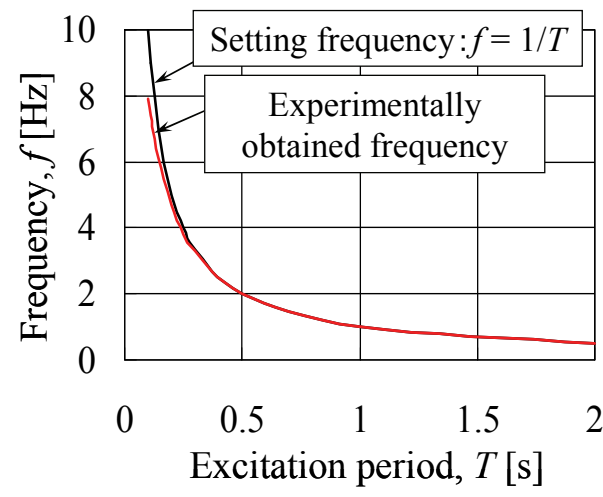

Fig. 14. Variation of the obtained excitation frequency versus the inputted excitation period.

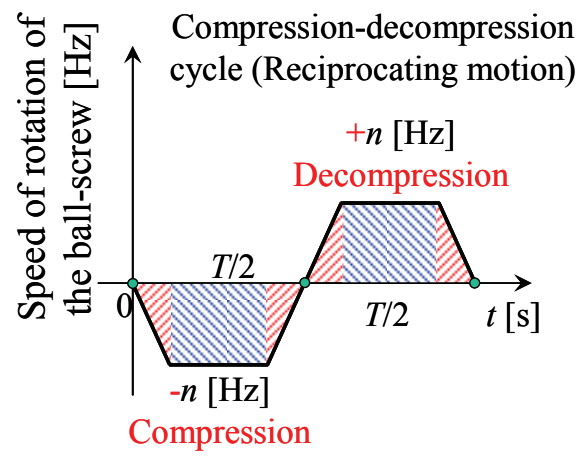

Fig. 15. Variation of the rotational speed of the ball-screw during one cycle of compression-decompression.

Such results can be explained by the fact that the piezoelectric stack is pre-compressed by a force of $6 \mathrm{kN}$.

From Fig. 14 one observes that the experimentally obtained excitation frequency is smaller than the desired frequency calculated as the inverse value of the inputted period of excitation, especially for frequencies exceeding 4 Hz. For example, as shown in Fig. 14, although the desired frequency is $10 \mathrm{~Hz}$, the actual frequency becomes $8 \mathrm{~Hz}$.

This can be explained by the trapezoidal shape of the diagram of variation of the rotational speed of the ball-screw during one cycle of compression-decompression (see Fig. 15).

In other words, although a rectangular diagram is desirable, since it is technically impossible to obtain sudden (infinite) acceleration and deceleration of the ball-screw, an inevitable time-lag is to be expected. However, such problem can be solved by correcting the inputted excitation period with the time-lag, in order to achieve during vibration experiments the exact desired value of the working frequency.

\section{Conclusions}

In this work, the energy harvestable from a colloidal vehicle suspension was evaluated. The following main conclusions were inferred:

1) Pressurizing the piezoelectric device along both axial and radial directions, a double amount of electrical power can be generated.

2) In the case of a classical vehicle suspension, pressure of the working fluid inside the cylinder of an oil damper or inside the air bag of a pneumatic suspension varies in the range of 0 to $10 \mathrm{MPa}$. However, in the case of a colloidal suspension, pressure inside the cylinder varies in the range of 20 to $60 \mathrm{MPa}$, i.e., the maximum pressure is 6 times higher, and the pressure variation range is 4 times wider. Thus, a substantially increased harvesting effect can be obtained, due to the fact that the electrical energy production proportionally varies with the second power of the pressure change.

3) Harvested electrical power proportionally varies with the number of piezoelectric devices electrically connected in parallel.

4) In order to achieve a mean generated voltage of 12 $\mathrm{V}$ (usual voltage of a battery equipping an automobile propelled by an internal combustion engine), the necessary thickness of the piezoelectric device is too small, and consequently, the proposed harvesting colloidal suspension is unlikely to be used in practical applications.

5) In order to achieve a mean generated voltage of 24 $\mathrm{V}$ (usual voltage of a battery equipping a truck propelled by an internal combustion engine), a mean generated voltage of 202 and $288 \mathrm{~V}$ (voltage of the main battery of a hybrid vehicle), the necessary thickness of the piezoelectric device $(l=0.1,0.7,1.0 \mathrm{~mm})$ can be technologically obtained in a relatively easy manner. Thus, the harvesting colloidal damper is likely to be used in such practical applications.

6) Although the harvestable power $(1-100 \mathrm{~W})$ is relatively modest, some possible applications such as, charge and recharge of a battery, or usage as electrical power source for the actuator of a fuel injection system can be considered.

7) Although piezoelectric effect cannot be obtained under tension loading for the traditional piezoelectric stacks, in the case of the proposed suspension, the piezoelectric stack is serially mounted together with a helical compression spring. Since the spring is pre-compressed by a force of $6 \mathrm{kN}$ such vehicle suspension is able to support both compressive and tensile loads. 
8) Experimentally obtained excitation frequency is smaller than the desired frequency calculated as the inverse value of the inputted period of excitation, especially for frequencies exceeding $4 \mathrm{~Hz}$. This can be explained by the trapezoidal shape of the diagram of variation of the rotational speed of the ball-screw during one cycle of compression-decompression, due to the technical impossibility to obtain sudden (infinite) acceleration and deceleration of the ball-screw. However, such problem can be solved by correcting the inputted excitation period with the time-lag.

\section{References}

(1) Allen Fuhs : "Hybrid Vehicles and the Future of Personal Transportation”, CRC Press, 2009

(2) Andreas Rau, Oliver Becker, and Erik Tonner : "Fuel Injection System and Method for Ascertaining a Needle Stroke Stop in a Fuel Injector", US patent 059021 A1, 2010

(3) Takeshi Okamura : "Multi-Layer Piezoelectric Element, Ejection Device having the Element and Fuel Ejection System", US patent 294853 A1, 2010

(4) Harry Zervos : "Energy Harvesting for Automotive Applications", IDTECHEX, pp. 1-19, 2011

(5) Hirofumi Inoue, Hiroaki Date, and Kazuo Ogawa: "Vehicle Suspension System", US patent 0230876 A1, 2010

(6) Atsuo Morikawa : "Hydraulic Suspension System able to Generate Electrical Power", Japanese patent 25901, 2011 (in Japanese)

(7) Hongbin Ren, Sizhong Chen, and Zhanzong Feng : "The Design and Simulation Analysis of Electromagnetic Energy Regenerative Suspension System", Proceedings of the FISITA World Automotive Congress, Vol. 7, pp. 601-610, 2012

(8) Shunsuke Kobayakawa : "Electromagnetic Induction Suspension System able to Generate Electrical Power and Associated Sensor to Extract the Vibration Information", Japanese patent 46791, 1995 (in Japanese)

(9) Teruhiko Kano : "Device able to Generate Electrical Power", Japanese patent 4359901, 2009 (in Japanese)

(10) Valentin Suciu : "Piezoelectric Colloidal Damper", Japanese patent 219900, 2012 (in Japanese)

(11) Valentin Suciu, and Ryouta Mouri : "Study on the Electric Power Generation using the Car Suspension System", JSME, Proceedings of the Symposium No. 128-3, pp. 19-20, 2012 (in Japanese)

(12) Valentin Suciu, and Kazuhiko Yaguchi : "Endurance
Tests on a Colloidal Damper Destined to Vehicle Suspension", Experimental Mechanics an International Journal, Vol. 49, pp. 383-393, 2009

(13) Valentin Suciu, Shingo Tani, and Kazuhiko Yaguchi : "On the Fatigue Fracture at Adsorption/Desorption of Water in/from Liquid-Repellent Nanoporous Silica", Acta Mechanica, Vol. 214, pp. 195-203, 2010

(14) Valentin Suciu, and Tsubasa Tobiishi : “Comfortableness Evaluation of an Automobile Equipped with Colloidal Suspensions", Journal of System Design and Dynamics, Vol. 6, No. 5, pp. 555-567, 2012 